\title{
On the Design of Morphing Airfoils using Spinal Structures
}

\author{
Narcis M. Ursache*, Andy J. Keane ${ }^{\dagger}$ and Neil W. Bressloff ${ }^{\ddagger}$ \\ University of Southampton, Southampton, SO17 1BJ, UK
}

\begin{abstract}
In this paper the design of spinal structures for the control of morphing airfoils is examined. The aim is to find structures that, when suitably loaded, can alter the aerodynamic shape of a cladding that forms the airfoil. Morphing through different cambered airfoils to achieve aerodynamic properties for different maneuvers is then possible by exploiting a range of incremental non-linear structural solutions. Further, by using structures that are acting in the post-buckling regime, it is possible to obtain significant changes in shape with only modest changes in applied load. The structure also presents enhanced aeroelastic properties. Results are formulated in terms of the aerodynamic properties of the morphed airfoils using a shape optimized beam as the spinal structure with fixed aerodynamic cladding.
\end{abstract}

\section{Introduction}

The availability of new technology and improved analytical tools has enlarged study possibilities for multi-structural systems. Smart aero-structures and compliant control surfaces have consequently become a potential way forward in the development of adaptive wings. Modern wing morphing concepts require a structure within a wing that can continuously change the shape of the wing in flight to alter the flow stream and achieve enhanced or changed aerodynamic properties, without the hinge contour discontinuity associated with articulated surfaces.

There is a great deal of current interest among aircraft designers in such shape control systems, primarily because engineers seek designs that have low radar signatures or are very quiet in operation. By removing hinged control surfaces roll performance and reversal speed can also be improved. ${ }^{1,2}$

Flapless, variable geometry airfoils are not a new idea. The original Wright Brothers Flyer used a "wing warping" concept to provide control, following developments with gliders. ${ }^{3}$ Since ailerons had not been invented at that stage, the brothers suggested that their approach would provide benefit in flying an aircraft. Their control system worked by pulling on a set of steel wires which twisted the wing tips relative to the rest of the wing.

Since the early 1980's, researchers have investigated the use of fully integrated smart structures for performance and shape control of deformable flight devices. In these, the wings become adaptive in the sense that they can change profile to adapt to flow conditions by controlled transitions from one airfoil shape to another. Deformable surfaces in conventional approaches can be replaced by micro-surface effectors (e.g., piezoelectric actuators), internal mechanical forces (e.g., piston-type actuators) or fluidic devices (i.e., synthetic jet actuators), offering great potential in controlling the baseline aerodynamic characteristics of the entire airfoil.

Schemes have been proposed to enhance flow conditions over wings by adapting the aerodynamic shapes through translation actuators placed in the rib stations span wise. ${ }^{2,4}$ Such approaches for morphing wing sections require an optimized distribution of forces and reliable and lightweight actuators. The investigation of synthetic jet actuators has been considered for manipulating flight control characteristics and flow separation $^{5,6}$ by interacting the streamlines with a jet generated by miniature surface-mounted devices, inducing an apparent change in shape. Highly compact actuators using smart materials have also provided a means of

*Research Student, CEDC, Univ. of Southampton, Highfield, Southampton, UK, SO17 1BJ, AIAA member.

$\dagger$ Professor of Computational Engineering, CEDC, Univ. of Southampton, Highfield, Southampton, UK, SO17 1BJ.

${ }^{\ddagger}$ Senior Research Fellow, CEDC, Univ. of Southampton, Highfield, Southampton, UK, SO17 1BJ. 
changing shapes and controlling structures, and mitigated some of the weight penalties of other approaches. Various schemes that induce strain in structures have been built using smart memory alloys (SMA's) and piezoelectric actuators, and used to adjust the thickness of airfoils to control flow separation, ${ }^{7,8}$ also to offer high precision operation and finite deformation in antenna reflectors. ${ }^{9,10}$ These approaches offer reliable alternatives to complex and heavy wing structure controllers, but they have limitations in terms of power and deformation. The use of smart structures such as compliant mechanisms also provides a viable approach to achieve local shape deformation of systems and a more limited number of actuators is then needed to control the strain energy transmitted to the deformable shape. ${ }^{11,12}$ For example, locally buckled skin structures have been considered for shape control of specific regions of airfoils by Natarajan et al. ${ }^{13}$

A number of these ideas have been tested in practical applications, for example the Mission Adaptive Wing ${ }^{14}$ and the Active Aeroelastic Wing (AAW). ${ }^{15}$ The AAW was used on an early version of the F-18 fighter to provide enhanced flight characteristics at supersonic speeds by controlling the induced strain in structures with piezoelectric actuators. The morphing concept also has significant applicability for Unmanned Air Vehicles (UAV's), to enhance their maneuverability and cope with complex-role missions with an inherent trade-off with regard to endurance and range (e.g., buckle-wing, ${ }^{16}$ torque-rod actuated wing ${ }^{17}$ ).

The primary focus of the current work is to develop new means of achieving deformable airfoils, by using the concept of spinal structures first introduced by Ursache et al. ${ }^{18}$ The methodology proposed in this paper involves both structural and aerodynamic optimization with respect to the geometry of the airfoils under different flow conditions. The aerodynamic features are defined by a four-digit NACA airfoil definition $^{\mathrm{a}}$, which is continuously deformed by the central spinal structure. The structural stability of the setup, including the post critical regime (i.e., post-buckling) is included in the design process to allow for large displacements (i.e., of the order of a camber of the airfoil) with modest changes in strain energy. A static aeroelastic study is also performed on the final optimum spinal structure to demonstrate the properties of the proposed design.

\section{Global Shape Control}

Wing morphing technology involves changing control surface shapes during flight to provide varying aerodynamic properties (i.e., for changes in mission or maneuver). The means of airfoil reshaping presented in the literature mainly focus on targeted local changes using a flexible outer skin (see for instance Ref. 11 and 13). A flexible outer skin is also adopted here, but in contrast to much of the work reported in the literature, the entire airfoil ship is altered. This global reshaping is achieved by distorting a slender internal spinal structure which is attached to a hyperelastic outer cladding that forms the aerodynamic surface of the morphed airfoil. Since each maneuver during flight may require a different camber configuration, the system proposed here morphs through a significant camber range using an incremental loading scheme. This allows a series of target aerodynamic shapes to be realized (in this study a set of NACA-four digit airfoils).

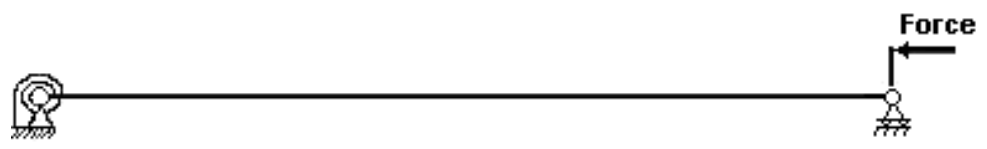

Figure 1. Structural set-up for morphing structural optimization.

In this paper, buckled strut structures are used to achieve global aerodynamic shape control. The spinal structural approach proposed here is a simply supported Euler strut subject to an eccentric load (i.e., to trigger the post-critical response), as depicted in figure 1. The eccentricity is exaggerated in the figure, to highlight the asymmetrical nature of the loading. The amplitude of the deflection that a regular strut takes up is intrinsically linked to the load function in a highly non-linear way, so that modest increases in load result in significant changes in shape (see figure 2). If, however, a strut with varying structural properties is used (for example varying lateral stiffness, material, etc.) the strut ceases to take up a simple shape when loaded (pure sine waves given by the first structural eigenmodes). By suitable choice of material

\footnotetext{
${ }^{a}$ Means to improve upon established implicit correlation between the deflected spinal structure and the theoretical airfoil surface are not provided in this paper, but it has been found that a hyperelastic material enables reactions on the strut with magnitude of an increment, which does not alter the accuracy of the optimum configuration
} 
properties, realistic camber profiles can be generated. If the spinal structure (i.e., airfoil camber shape) is then connected to a semi-rigid pre-tensioned elastic skin via a flexible foam core, this affords a means of airfoil section shape control (here a base-line un-cambered four-digit NACA thickness definition is chosen for its analytical simplicity).

The selection of the varying geometrical properties is here accomplished via an inverse design procedure whereby an optimizer is used to control the structural properties and end loads. Working in the postbuckling regime, the load-carrying capacity of slender structures is significantly affected by the presence of imperfections. This can be seen in figure 2, where an irregular column, loaded from its unstrained state will give a smooth equilibrium path with no instability. The response of the structure will grow rapidly and continuously until the critical load is reached and the transition into the post-critical state is smooth. The stiffness of the system tends to a singular state as the axial force approaches the critical Euler load, accelerated by the second-order effects in the total strain definition of the initial imperfection. An additional restraint (i.e., a rotational spring) is here applied to control the gradient of the lateral deformation towards the pinned end, and, by augmenting the stiffness of the structure, has a significant effect on the pre and post-buckle pattern response. ${ }^{19}$

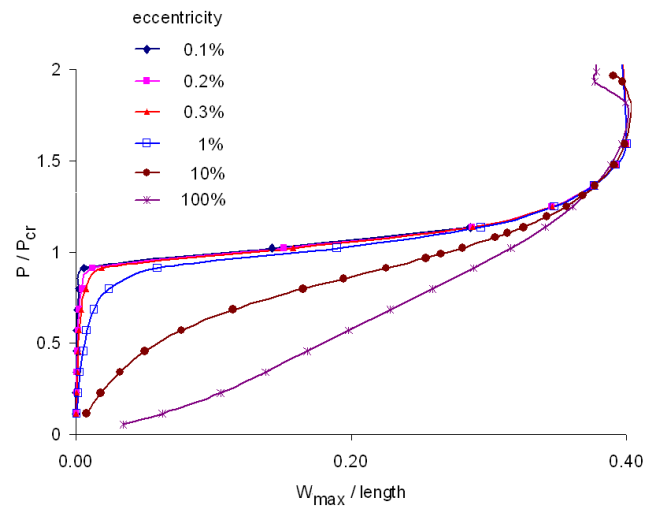

Figure 2. Sensitivity of a regular strut to eccentricity of loading.

Since the aim during operation is to move the spinal structure smoothly between a series of pre-defined camber line shapes it is necessary to find structures that deform through such shapes during their postbuckling behavior - this is, of course, more difficult than finding a strut that produces a single desired camber line at one fixed load value. To solve this problem a non-linear finite element analysis is performed using an incremental loading scheme, ${ }^{20,21}$ with a static equilibrium being obtained after each load increment. This allows the full range of shapes possible for any particular material layout to be assessed.

Then, during design, optimizers are used to try and match these shapes to a series of NACA camber lines by adjusting the properties of the strut. Inevitably such matches cannot be perfect throughout the range of loading but surprisingly good agreement can be achieved so that the resulting sequences of wing morphs are remarkable close to the desired airfoil shapes. The quality of these shape sequences are here assessed using a full potential Computational Fluid Dynamics (CFD) simulation and compared to those of the target airfoils. Even when there are slight differences between the shapes considered, the differences in the resulting pressure distributions are modest. To minimize such differences, a second optimization process can be entered which seeks to further refine the structural design by minimizing differences between the desired and achieved pressure distributions, requiring linked CFD and structural analyses.

\section{Optimization}

Optimization tools provide a means to achieve better devices during a shape design process, involving strategies such as direct analysis or an inverse approach. In the first case, one studies the effects of parameter variations via an objective function which is formulated with respect to some target performance metric (such as low drag) with constraints that can be structural in nature or aerodynamically related. The inverse approach works towards a given shape by attempting to push some derived characteristic towards a desired configuration. The derived characteristic is usually specified as a field variable (e.g., static pressure or 
freestream flow) that is, a priori, known to yield desirable performance. Inverse methods are useful when designing systems with specific characteristics, as undesirable physical effects such as shocks, or flaws in shapes can be explicitly avoided.

A large number of papers dealing with optimal design problems via inverse strategies can be found in the literature. A typical goal in inverse structural design is achieving meaningful shapes that conform to specified boundary conditions and fulfill functions such as structural integrity with acceptable performance (e.g., acceptable nominal stress).

A significant body of work on the stability of structures subject to displacement and stress constraints has been developed since the early 1970s, as noted in a survey by Haftka and Prasad. ${ }^{22}$ This has been followed more recently by studies on optimality criteria with displacement and stress constraints using more powerful numerical algorithms, ${ }^{23}$ from maximizing the minimum critical load or natural frequencies ${ }^{24}$ to stability of imperfection-sensitive structures in the post-critical regime. ${ }^{25}$ The optimization of geometrically non-linear structures is limited with respect to shape and boundary conditions and requires a good understanding of the target structural shape. A general methodology for inverse design for simple structures has been proposed by Bernitsas et al. ${ }^{26}$ Their goal was to achieve improved structures (e.g., reduced vibration amplitudes), subject to various dynamic excitations.

This work pursues two threads towards global optimization, by providing aerodynamic enhancement during morphing, which is directly linked to the structural shapes achieved. The primary goal is the novel control of the aerodynamic NACA-based parameterized shapes, driven by a stochastic parent-based search followed by a gradient-based search on the structural problem (i.e., Genetic Algorithm ${ }^{27}$ and Dynamic Hill Climbing, ${ }^{28}$ both taken from the Options design exploration toolkit $\left.{ }^{\mathrm{b}}\right)$. The geometrically non-linear structure is optimized with respect to its stability, with failure criteria included in the optimization function to avoid instabilities in non-linear response, such as snap-through or snap-down, due to widely varying flexural stiffness and the end rotational restraint. These instabilities are checked against the load proportionality factor which can exhibit one or more limits and/or turning points before achieving the final cambers of interest. The structural optimization process is driven by a search engine through the design variables, chosen from within a set of available values, such that the objective function is minimized or maximized, with failure criteria formulated with respect to the constraints. This process requires some care when setting up but can be directly handled using commercial FEA codes (here Abaqus@).

\section{A. Hybrid Search}

The non-linear problem stated here is tackled by a global load control algorithm and the convergence of the solution is achieved by breaking the simulation into a number of increments. Here the optimization problem can be stated for global search in terms of the deflected shape of the strut at a series of loading increments as follows:

$$
\begin{aligned}
& \text { Minimize } f_{1}(x)=\frac{1}{n_{i}} \sum_{i=1}^{n_{i}}\left\|w(x)^{t}-w(x)^{c}\right\|^{i} \\
& \text { Subject to }{ }^{1} g_{i}(x)=\left.\max \right|_{j} w_{i j}^{c}-a_{2} \leq 0 \\
& { }^{2} g_{i}(x)=a_{1}-\left.\max \right|_{j} w_{i j}^{c} \leq 0 \\
& x \in X, \forall j \in\left\{1, \ldots, n_{p}\right\}, \forall i \in\left\{1, \ldots, n_{i}\right\}
\end{aligned}
$$

where $X=\left\{x \in \Re^{n} \mid x_{k}^{\min } \leq x_{k} \leq x_{k}^{\max }, k=1, \ldots, n_{v}\right\}$ with $x_{k}^{\min }$ and $x_{k}^{\max }$ bounds on the $n_{v}$ structural variables set by the user; $w_{i j}$ are the deflections at load increment $i$ and structural location $j$, and $a \in\left\{a_{1}, a_{2}\right\}$ define the lower and upper displacement constraints at each load increment, with a maximum number of increments $n_{i}$, for each airfoil defined at $n_{p}$ structural grid points. Note that $w(x)^{t}$ are chosen from the target camber lines by selecting cambers that have similar overall maximum deflections to those arising at any specific load increment $i$ - this further speeds up the design process since it is then no longer necessary to know the specific control force needed to achieve a given shape. For the structures considered here which

\footnotetext{
${ }^{\mathrm{b}} \mathrm{http}$ ///www.soton.ac.uk/ ajk/options/welcome.html
} 
are of $1 \mathrm{~m}$ length and $8 \mathrm{~mm}$ width, typical end forces are in the range $40-85 \mathrm{~N}$, with variations of less than $2 \mathrm{~N}$ being needed to deflect the camber from 5 to 10\%, typically around 1\% changes in end force being needed.

A high-fidelity optimization for feasible and enhanced structures requires a shape parameterization concept. The aim of most parameterizations is to use mathematical methods to describe curves which can be flexible enough to represent a wide range of shapes, which can be easily controlled, increasing the number of potential solutions. Robust geometry parameterizations techniques have been available in the literature since the late 1970s, as comprehensively described in Samareh's survey, ${ }^{29}$ along with their shortcomings. In this paper, because of the limitations of space, the results based on Discrete Approach (DA) are described, although a NURBS-based parameterization was also tested, leading to similar results.

To allow for a wide range of possible shapes, a subset of the finite element grid points coordinates in the structural model are used to define the regions for application of design variables. Therefore the crosssectional areas of twelve sections of the spinal beam are varied by parameterizing the thickness distribution along its length (as depicted in figure 3 - note that the width of the spine is held fixed at $8 \mathrm{~mm}$ ). The resulting design is used as to achieve aerodynamic shapes by applying a fixed NACA-based $12 \%$ thickness distribution to yield the morphed shape. Although the optimization problem may be readily setup in this way, the resulting structural geometry lacks smoothness and is somewhat impractical to manufacture (its deflected shape and that of the airfoil, of course, retain second order smoothness throughout) .

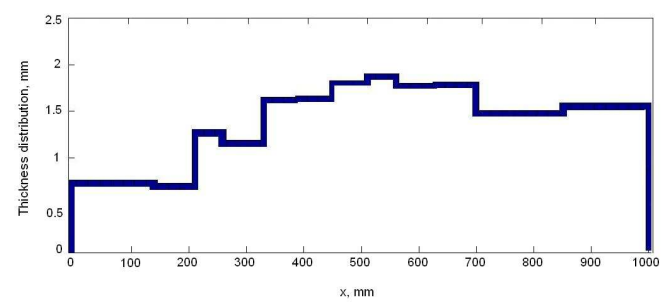

Figure 3. Morphing-optimized beam - semi thickness, global solution.

Typical cambered shapes resulting from the global scheme (i.e, at $5 \%$ and $10 \%$ camber) are shown in figure 4, along with the target NACA sections used in the inverse design.
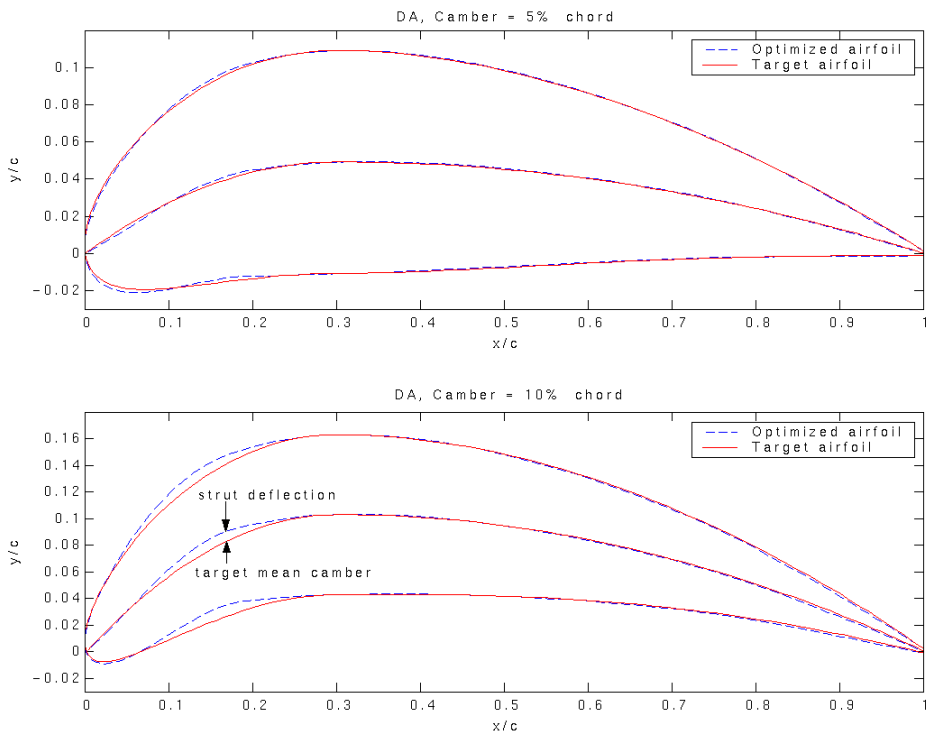

Figure 4. Target and computed airfoils for morphing optimized beam.

The similarity in shapes obtained with DA and NURBS-based parameterizations (not presented in this paper) suggests that the structural solution to the camber matching is not highly multi modal and that 
either form presents a good basis from which to make further refinement.

To improve upon the structural solution achieved with the global search, a design refinement in terms of aerodynamic properties of morphing airfoils is performed using a CFD-based inverse optimization with a gradient-based engine and this problem can be stated as:

$$
\text { Minimize } f_{2}(x)=\left\|C_{p}(x)^{t}-C_{p}(x)^{c}\right\|^{i}
$$

$$
\text { Subject to }{ }^{1} g_{i}(x) \text { and }{ }^{2} g_{i}(x) \text {. }
$$

where increment $i$ represents the structural solution corresponding to an airfoil with maximum camber of $10 \%$ chord.

\section{B. Aerodynamic Performance of Morphing Airfoils}

As the shape of the airfoil changes, the flow around it also changes. This leads to an altered pressure distribution, which, in turn, modifies the aerodynamic properties of the model. A two-dimensional viscous coupled finite difference code that solves the full potential equations, VGK, ${ }^{30}$ is used here to predict the aerodynamic properties of the morphing airfoils. The convergence of the aerodynamic iterative solution depends upon the flow characteristics (e.g., Mach number, freestream incidence, Reynolds number, transition locations). VGK provides good accuracy for flows with weak shocks and attached boundary layers and fair predictions of local and overall parameters when the upstream Mach number just before weak shocks does not exceed 1.3 with separation of the boundary-layer.
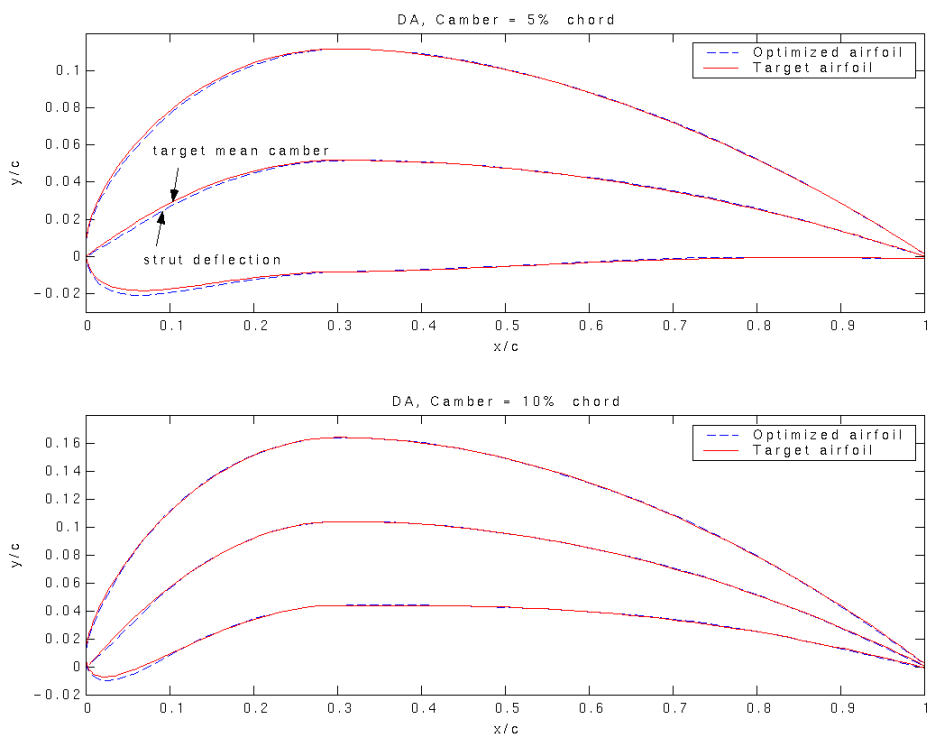

Figure 5. Target and computed airfoils for morphing-optimized beam after CFD-based optimization.

The refining optimization process is driven by a dynamic hill-climbing, gradient-based method, using as a starting solution the best from previous optimization (i.e., the best design after 100 generations of the GA, each of 50 members is taken to be initial optimum and analysed using CFD for subsequent fine tuning for fixed flow condition at $M=0.5$ and $\alpha=2 \mathrm{deg}$ ). Here 1600 design evaluations are used and this further improves the geometric matching (see figure 5).

A standard setup in terms of Mach number and angle of incidence has been built for the target and parameterized airfoils. This covers Mach numbers between 0.4 and 0.8 , freestream incidence between -5 and +10 degrees, and is for a fixed Reynolds number of $5 * 10^{6}$, transition location 0.03 and shapes with between $2 \%$ and $15 \%$ camber to chord ratio. Flows with these parameters have been studied for the both computed and 

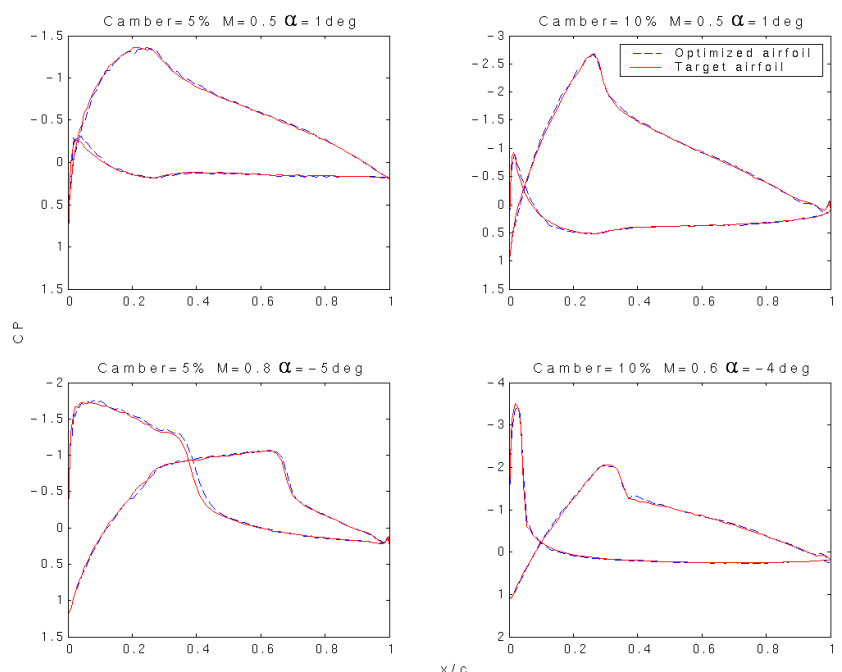

Figure 6. Refined distribution for morphed airfoils, under mild and extreme computed flows.

target airfoils in order to assess their agreement in terms of pressure distributions (see figure 6). Clearly, the pressure distributions are sensitive to changes in geometry, flow parameters and boundary layer growth and the flow can readily degenerate into a weak shock for higher cambers and transonic conditions, contributing wave drag. Both $5 \%$ and $10 \%$ cambered airfoils show a very good agreement in terms of pressure distribution. As the camber increases, the tendency to upper surface boundary-layer separation becomes more significant and also weak shocks occur. The drag polar trends are similar across a wide range of incidences at $\mathrm{M}=0.5$, yielding a close agreement for $5 \%$ camber, with a maximum 0.09 drag count difference between the two airfoils for extreme incidences studied, whereas the $10 \%$ target airfoil shows locally a higher drag with a magnitude of 0.01 (see figure 7). Of course, it would be possible to carry out the entire optimization process using the combined structural and CFD analysis throughout, but this would be considerably more costly than the two stage process used here.
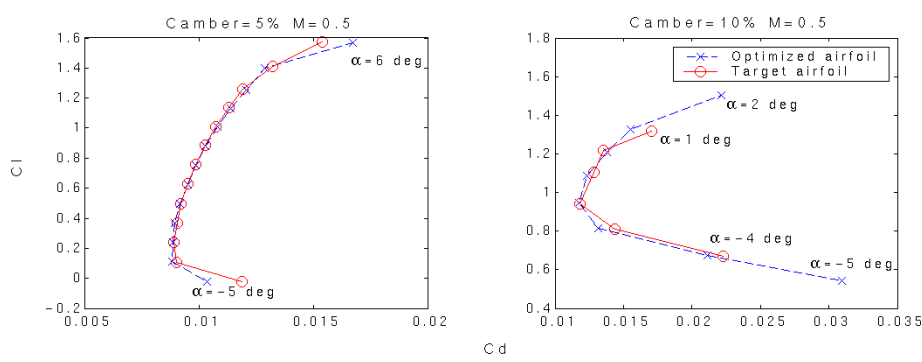

Figure 7. Refined drag polars for morphed airfoils.

\section{Static Aeroelastic Study of Morphing Airfoils}

To illustrate the impact on the optimum morphing shape of the aerodynamic loading, a canonical study consisting of a $5 \%$ cambered airfoil under moderate flow conditions with $M=0.5$ and $\alpha=1$ deg has been conducted. Here, a loosely coupled and modular method for the static aeroelastic study is performed, since time-accurate solutions are not considered. In $\mathrm{VGK}^{30}$ the infinite region outside the aerofoil is mapped conformally onto the inside of a circle and the computational grid utilized by the finite-difference method 
is built upon radial lines and concentric circles. A fine CFD grid is utilized ab initio in order to keep the accuracy of the solution for any small geometry changes. Therefore, the aeroelastic solution is based upon the boundary interface corresponding to a fixed CFD grid and an iteratively updated displacement field (i.e., a pressure field is obtained from a rigid steady state CFD solution and is mapped onto the boundary-fitted structural grid with impact on structural strength computed by the FEM solver).

Static aeroelasticity requires geometry conservation through the mapping of the conservative aerodynamic loads, which becomes intrinsic when a relaxation factor $\beta$ is used (see equation (3)) to adjust the highly oscillatory trend of the convergence of the solution, as suggested in literature (see for instance Cai and Liu ${ }^{31}$ or Alonso $e t a l^{32}$ ). The relaxation procedure is used here to augment the pressure field with the current $f^{n}$ and previous one $f^{n-1}$ and the convergence history is controlled by the weighting factor (i.e., $\beta=0.4$ ).

$$
f^{n}=f^{n-1}+\beta\left(f^{n}-f^{n-1}\right)
$$

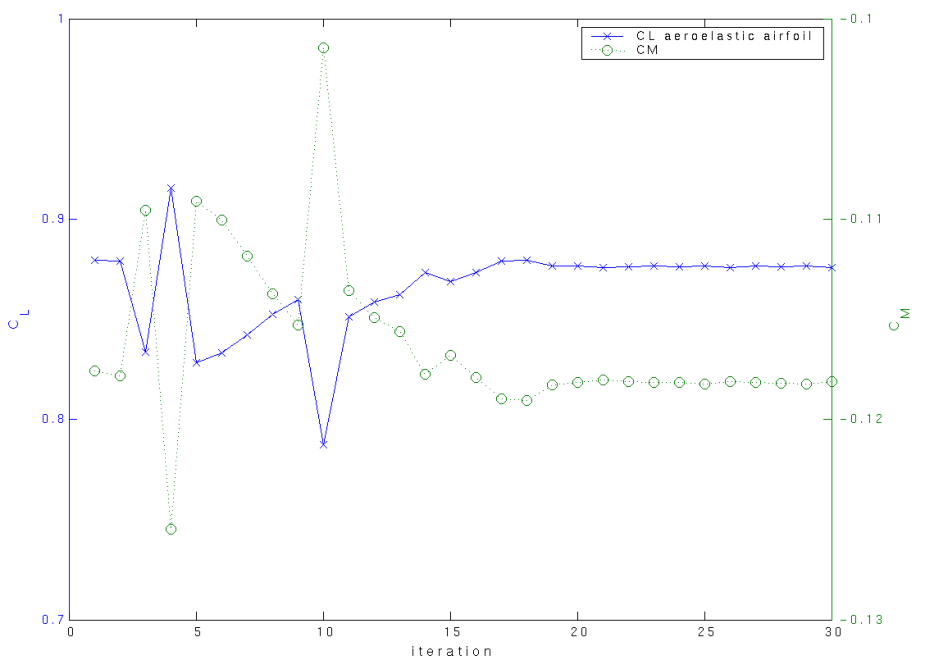

Figure 8. Aeroelastic stability, $\beta=0.4$.

The convergence history in terms of lift and pitching moment coefficients in figure 8 , shows large oscillations due to the incremental-iterative FEM procedure used. The difference between two consecutive incremental solutions can be large and then the minimum norm of the desired camber and the surrounding solutions is chosen, which may lead to oscillations in aerodynamic properties during the design cycle due to the somewhat large camber variations (the variation is dictated by the non-linear solution and has a magnitude of an increment). Structural instabilities may also occur, in which case the relaxation factor is augmented by a one percentage to add a larger perturbation to the system for a stable solution. The convergence history also shows that the aeroelastic solution (obtained for a relaxation $0.4<\beta<0.46$ ) is slightly augmented than the rigid steady state one with maximum one percentage, which shows that the airfoil is stiff enough to preserve its optimized rigid state properties (see figure 9 for the aeroelastic pressure distribution and the resultant airfoils in figure 10).

\section{Conclusions}

The focus of this research has been to find a means to alter the global aerodynamic performance of an airfoil by controlling its deformable shape. The aim was to find structures that, when suitably loaded, can be used to alter the flow on a cladding that forms the airfoil. Further, by using structures that are acting in the post-buckling regime, it is possible to obtain significant changes in shape with very modest changes in applied load. A very good agreement between airfoils constructed around a deflected strut and target NACA-four digit airfoils has been obtained in terms of aerodynamic performance. It is clear that, by making use of 


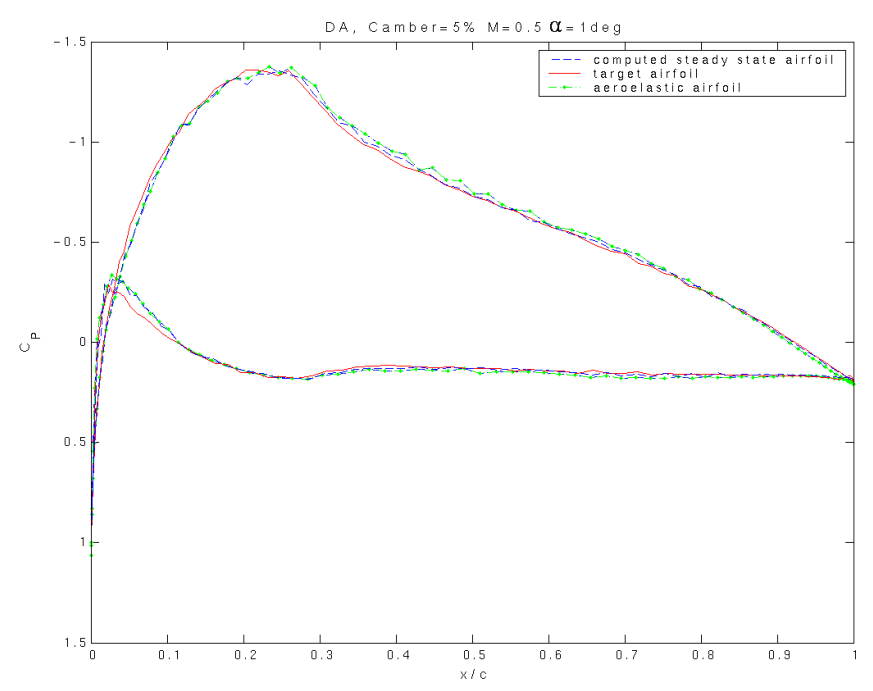

Figure 9. Aeroelastic pressure distributions, $\beta=0.4$.

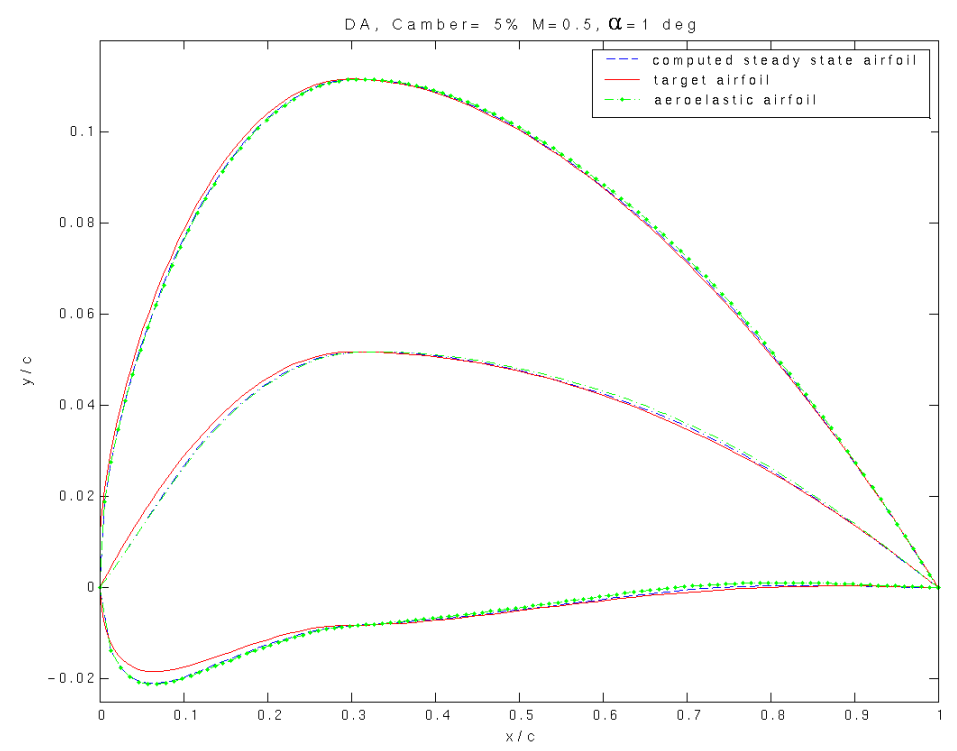

Figure 10. Aeroelastic airfoils, $\beta=0.4$.

non-linear structural responses, camber control of deformable airfoils can be achieved by using a carefully designed pre-loaded internal spinal structure that moves through the desired shape changes under the control of a single actuator, delivering aerodynamic characteristics that match a set of pre-specified target shapes and also give good aeroelastic properties.

\section{Acknowledgments}

This research was supported by a grant from the Faculty of Engineering, Science and Mathematics at the University of Southampton. 


\section{References}

${ }^{1}$ Khot, N. S., "Deformation of a Flexible Wing using an Actuating System for a Rolling Maneuver without Ailerons", AIAA-98-1802.

${ }^{2}$ Gern, F. H., Inman, D. J., and Kapania, R. K., "Structural and Aeroelastic Modeling of General Planform Wings With Morphing Airfoils", AIAA Journal, Vol. 40, No. 4, April 2002, pp. 628-637.

${ }^{3}$ Anderson, J.D., Introduction to Flight, McGraw-Hill Book Company, New York, 1978.

${ }^{4}$ Austin, F., Rossi, M. J., Van Nostrand, W., Gareth, K., and Jameson, A., "Static Shape Control for Adaptive Wings", AIA A Journal, Vol. 32, No. 9, Sept. 1994, pp. 1985-1901.

${ }^{5}$ Chatlynne, E, Rumingny, N., Amitay, M., and Glezer, A., "Virtual Aero-Shaping of a Clark-Y Airfoil Using Synthetic Jet Actuators", AIAA 2000-0732, 2000.

${ }^{6}$ Chen, F. J., and Beeler, G. B., "Virtual Shaping of a Two-dimensional NACA 0015 Airfoil Using Synthetic Jet Actuator", AIAA 2002-3273.

${ }^{7}$ Seifert, A., Eliahu, S., Greenblatt, D., and Wygnanski, I., "Use of Piezoelectric Actuators for Airfoil Separation Control", AIAA Journal, Vol. 36, No. 8, Aug. 1998, pp. 1535-1536.

${ }^{8}$ Munday, D. and Jacob, J., "Active Control of Separation on a Wing with Conformal Camber", AIAA Aerospace Sciences Meeting and Exhibit, AIAA 2001-0293, Jan.8-11, 2001/Reno, NV.

${ }^{9}$ Balas, M. J., "Optimal Quasi-Static Shape Control for Large Aerospace Antennae", Journal of Optimization Theory and Applications, Vol. 46, No. 2, 1985, pp. 153-170.

${ }^{10}$ Yoon, H. S., Washington, G., and Theunissen, W. H., "Analysis and Design of Doubly Curved Piezoelectric Strip-Actuated Aperature Antennas", IEEE Transactions on Antennas and Propagation, Vol. 48, No. 5, May 2000, pp. 755-763.

${ }^{11}$ Saggere, L., and Kota, Sridhar, "Static Shape Control of Smart Structures Using Compliant Mechanisms", AIAA Journal, Vol. 37, No. 5, May 1999, pp. 572-578.

${ }^{12} \mathrm{Lu}, \mathrm{K}$. J., and Kota, S., "Synthesis of Shape Morphing Compliant Mechanisms Using a Load Path Representation Method", Smart Structures and Materials 2003: Modeling, Signal Processing and Control, Proceedings of SPIE, edited by R. C. Smith, Vol. 5049, 2003, pp. 337-348.

${ }^{13}$ Natarajan, A., Kapania, R. K., and Inman, D. J., "Aeroelastic Optimization of Adaptive Bumps for Yaw Control", Journal of Aircraft, Vol. 41, No. 1, Jan-Feb, 2004, pp. 175-185.

${ }^{14}$ Scott, W., "Performance Gains Confirmed in Mission Adaptive Wing Tests", Aviation Week and Space Technology, Vol. 129, No. 77, 1988, p.77.

${ }^{15}$ Pendleton, E., Bessette, D., Field, P., Miller, G., Griffin, K., "Active Aeroelastic Wing Flight Research Program: Technical Program and Model Analytical Development", Journal of Aircraft, Vol. 37, No. 4, July-Aug. 2000, pp. 554-561.

${ }^{16}$ Gano, S. E., Renaud, J. E., Batill, S. M., and Tovar, A., "Shape Optimization for Conforming Airfoils", Proceedings of the $44^{\text {th }}$ AIAA/ASME/ASCE/AHS Structures, Structural Dynamics and Materials Conference, AIAA 2003-1579, Norfolk, VA, April 7-10, 2003.

${ }^{17}$ Abdulrahim, M., Garcis, H., and Lind, R., "Flight Characteristic of Shaping the Membrane Wing of a Micro Air Vehicle", Journal of Aircraft, Vol. 42, No. 1, Jan-Feb 2005, pp. 131-137.

${ }^{18}$ Ursache, N. M., Bressloff, N. W., and Keane, A. J., "The Design of Post-Buckled Spinal Structures for Airfoil Shape Control Using Optimization Methods", Proc. of $5^{\text {th }}$ ASMO UK/ISSMO Conf. on Engineering Design Optimization, July, 2004. ${ }^{19}$ Stein, M., "The Phenomenon of Change in Buckle Pattern in Elastic Structures", NASA TR R-39, 1959.

${ }^{20}$ Crisfield, M. A., Non-linear Finite Element Analysis of Solids and Structures, Vol. 1, John Wiley \& Sons Ltd, England, 1997.

${ }^{21}$ Wempner, G. A., "Discrete Approximations Related to Nonlinear Theory of Solids", International Journal of Solids and Structures, Vol. 7, No. 11, Nov. 1971, pp. 1581-1591.

${ }^{22}$ Haftka, R. T., Prasad, B., "Optimum Structural Design with Plate Bending Elements - A Survey", AIAA Journal, No. 19, 1981, pp. 517-522.

${ }^{23}$ Zhou, M., Gu, Y. X., and Rozvany, G. I. N., "Application of DCOC method to Plates and Shells", Proceedings of the First World Congress of Structural and Multidisciplinary Optimization, edited by N. Olhoff and G. N. Rozvany, Germany, 1995, pp. 25-32.

${ }^{24}$ Olhoff, N., Krog, L. A., and Lund, E., "Optimization of Multimodal Eigenvalues", Proceedings of the First World Congress of Structural and Multidisciplinary Optimization, edited by N. Olhoff and G. N. Rozvany, Germany, 1995, pp. 701-708.

${ }^{25}$ Bochenek, B. "On Postbuckling Constraints in Structural Optimization Against Instability", Proceedings of the First World Congress of Structural and Multidisciplinary Optimization, edited by N. Olhoff and G. N. Rozvany, Germany, 1995, pp. 717-724.

${ }^{26}$ Bernitsas, M. M., and Suryatama, D., "Structural Redesign by Large Admissible Perturbations with Static Mode Compensation", ASME Trans, Journal of Offshore Mechanics and Arctic Engineering, 1999, Vol. 121, No. 1, pp.39-46.

${ }^{27}$ Holland, J.H., Adaptation in Natural and Artificial Systems: an Introductory Analysis With Applications to Biology, Control and Artificial Intelligence, The University of Michigan Press, Ann Arbour, MI, 1975.

${ }^{28}$ Yuret, D. and de la Maza, M., "Dynamic Hill Climbing: Overcoming the Limitations of Optimization techniques", Proceedings of the $2^{\text {nd }}$ Turkish Symposium on AI and ANNI, 1993.

${ }^{29}$ Samareh, J.A., "Survey of Shape Parameterization Techniques for High-Fidelity Multidisciplinary Shape Optimization", AIAA Journal, Vol. 39, No. 5, 2001, pp. 877-883.

${ }^{30}$ DERA, Farnborough, VGK Method for Two-Dimensional Aerofoil Sections, Ver. 2.0, ESDU 96028, ESDU $96029,1997$.

${ }^{31}$ Cai, J., and Liu L., "Static Aero-elastic Computation with a Coupled CFD and CSD Method", AIAA 01-0717, 2001.

${ }^{32}$ Alonso, J. J., LeGresley P., van det Weide E., Martins J. R., and Reuther J. J., "pyMDO: A framework for High-Fidelity Multi-Disciplinary Optimization", AIAA 2004-4480, 2004.

10 of 10 